\title{
Kératokystes des maxillaires : discordance entre les diagnostics clinique, radiologique et anatomopathologique. A propos de deux cas
}

\author{
Simohammed Bouzoubaa*, Mohamed Mokrane Khazana, Ihsane Ben Yahya \\ Service d'Odontologie chirurgicale, CCTD, Casablanca, Maroc
}

(Reçu le 28 janvier 2013, accepté le 18 février 2013)

Mots clés :
kératokyste
parakératosique /
kératokyste
orthokératosique /
kyste inflammatoire /
kyste dentigère

Key words: parakeratosic keratocyst / orthokeratosic keratocyst / dentigerous cyst / inflammatory cyst

\begin{abstract}
Résumé - Les kératokystes odontogènes des maxillaires se développent à partir de la lame dentaire, ou de ses vestiges, et de l'assise basale de l'épithélium buccal. Ils surviennent surtout lors de la deuxième et la troisième décades. Ils sont plus fréquents chez l'homme que chez la femme, et ils siègent préférentiellement à la mandibule (65\% à $80 \%$ des cas), dans la région de l'angle mandibulaire.

Ce travail rapporte deux cas de kératokystes. L'un est un kératokyste maxillaire évoquant, à l'examen clinique et radiologique, un kyste inflammatoire en raison de ses rapports avec 11, 21 et 22 qui sont nécrosées. L'autre est un kératokyste mandibulaire évoquant un kyste dentigère car il est associé à une 43 incluse.

Ces 2 cas permettent d'illustrer la difficulté de diagnostic à partir des éléments cliniques et radiologiques et d'insister sur l'intérêt de l'examen anatomopathologique.
\end{abstract}

\begin{abstract}
Keratocysts of the jaws: discordance between clinical, radiological and pathological diagnosis. Two cases report. Odontogenic keratocysts of the jaws are derived from the dental lamina, or its remnants, and the basal layer of the oral epithelium. They occur often during the second and third decades. They are more common in men than in women, and they sit preferentially in the mandible (65\% to $80 \%$ of cases), in the vicinity of the mandibular angle.

We report two cases of keratocysts. The first case is a maxillary keratocyst referring, to the clinical and radiological examination, an inflammatory cyst because its relations with the 11, 21 and 22 necrotic. The second case is a mandibular keratocyst evoking a dentigerous cyst because it is associated with the 43 included.

Through these two cases, we want to show the difficulty of diagnosis based on clinical and radiological aspect and to insist on the importance of histological examination.
\end{abstract}

Les kystes des maxillaires sont des lésions intraosseuses, le plus souvent d'origine odontogène, possédant alors une structure histologique particulière : la cavité kystique est bordée par un épithélium, kératinisé ou non, et son contenu est liquide, semi-liquide ou solide.

Le diagnostic de ces lésions n'est pas toujours facile, même si elles présentent des caractères cliniques et radiologiques et des particularités propres à chaque type de kyste. Le diagnostic basé sur l'expérience clinique peut induire en erreur, seul le diagnostic anatomopathologique peut assurer le diagnostic de certitude.

Sur le plan thérapeutique, la chirurgie reste de règle mais le pronostic diffère d'une lésion à l'autre. $1^{\text {er }}$ cas

Une femme, âgée de 29 ans, est venue consulter pour une tuméfaction naso-génienne gauche, haute, indolore, générant une asymétrie faciale depuis 1 an (Fig. 1). L'examen clinique révélait une tuméfaction de consistance ferme, bien limitée et non douloureuse, et l'absence d'adénopathies cervico-faciales. L'examen endobuccal montrait un comblement du vestibule supérieur para-médian gauche et une tuméfaction palatine antérieure gauche, avec une muqueuse de recouvrement d'aspect normal. Les dents antérieures étaient déplacées. À la palpation, on notait une soufflure des tables vestibulaire et palatine. Les tests de vitalité étaient négatifs pour 11, 21 et 22 (Fig. 2a et 2b).

\footnotetext{
*Correspondance : simobz@hotmail.com
} 


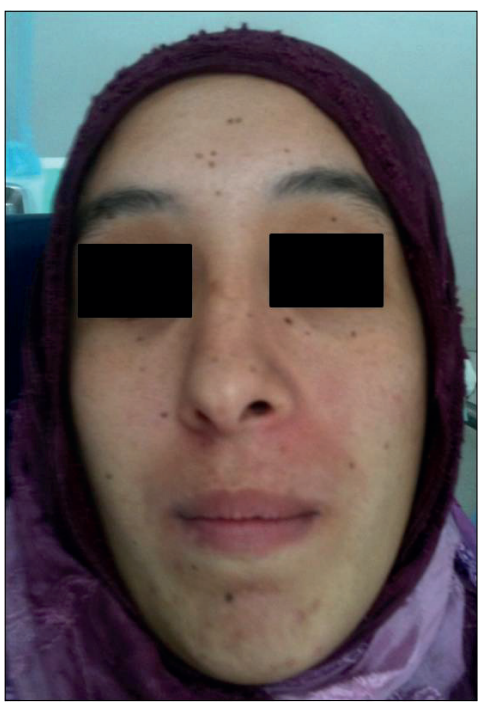

Fig. 1. Vue exobuccale : tuméfaction génienne haute gauche. Fig. 1. Exobuccal view: high left maxillary swelling.

L'orthopantomogramme montrait une image radioclaire d'allure kystique, bien limitée, qui s'étendait de la 12 à la 24 (Fig. 3). Le Dentascan précisait que cette lésion entrainait une lyse des corticales interne et externe et qu'elle présentait des rapports intimes avec les fosses nasales et le sinus maxillaire gauche (Fig. 4a et $4 b$ ). L'ensemble de ces éléments faisait évoquer un kyste inflammatoire.

La prise en charge de cette patiente a consisté à réaliser un traitement canalaire sur 11, 21 et 22 et une kystectomie (Fig. 5). L'examen anatomopathologique a conclu à un kératokyste type parakératosique (Fig. 6). Au $3^{\mathrm{e}}$ mois postopératoire, le contrôle clinique montrait une bonne cicatrisation muqueuse; au $12^{\mathrm{e}}$ mois postopératoire, le contrôle radiologique montrait un début de cicatrisation osseuse (Fig. 7 et 8).

\section{$2^{e}$ cas}

Un homme, âgé de 35 ans, est venu consulter pour une tuméfaction génienne droite, basse, indolore, générant une asymétrie faciale depuis 2 ans (Fig. 9). À l'examen exobuccal, la tuméfaction bien limitée et douloureuse à la palpation avait une consistance ferme. Il n'y avait pas d'adénopathies cervico-faciales ni de signe de Vincent. À l'examen endobuccal, on observait un comblement vestibulaire en regard de 44, 45 et 46 , avec une muqueuse de recouvrement d'aspect normal (Fig. 10) . À la palpation, la tuméfaction était molle. 44 et 45 étaient linguo-versées et le test de vitalité positif sur 44,45 et 46.

L'orthopantomogramme montrait une image radioclaire bien limitée qui refoulait la 43 incluse (Fig. 11). Le Dentascan confirmait qu'il s'agissait bien d'une image hypodense, bien limitée, qui soufflait la corticale externe et qui refoulait le nerf
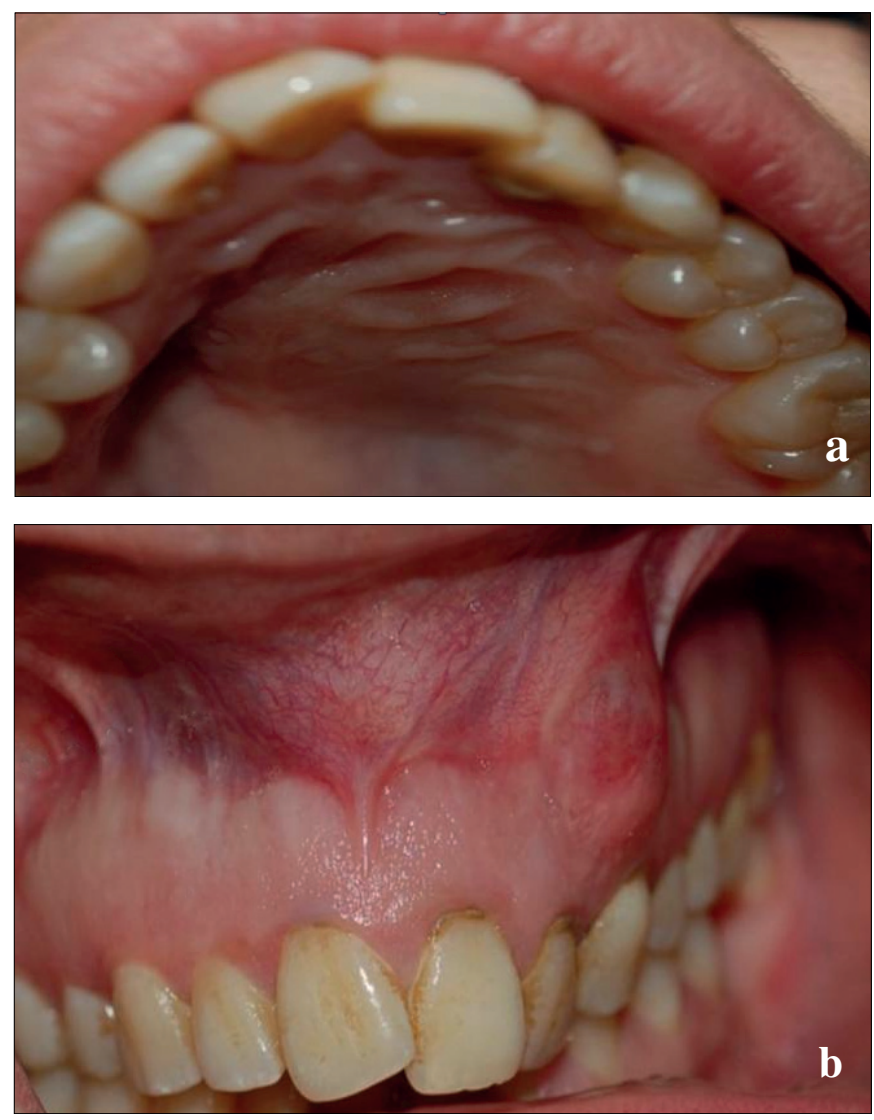

Fig. 2 a et 2 b. Vue endobuccale : comblement vestibulaire et tuméfaction palatine en regard de 21,22 et 23 qui sont en palatoversion.

Fig. $2 a$ and $2 b$. Intraoral view: vestibular and palatine swellings near to the 21, 22 and 23 displaced in the palate position.

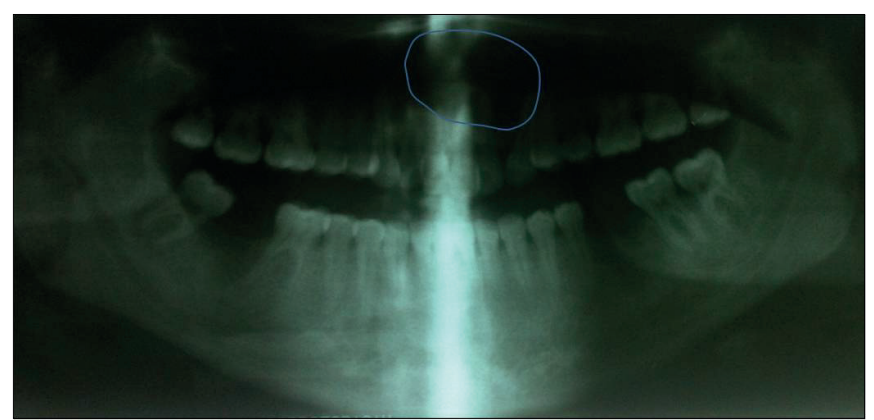

Fig. 3. Radiographie panoramique : image radioclaire, d'aspect kystique, bien limitée, allant de 12 à 24 .

Fig. 3. Panoramic radiograph: image of radiolucent cystic appearance, well limited, extended of 12 to 24.

alvéolaire inférieur vers le bas. Cette image était associée à une rhizalyse sur 44,45 et 46 (Fig. $12 \mathrm{a}$ et $12 \mathrm{~b}$ ). L'ensemble des éléments cliniques et radiologiques faisaient évoquer un kyste dentigère. 

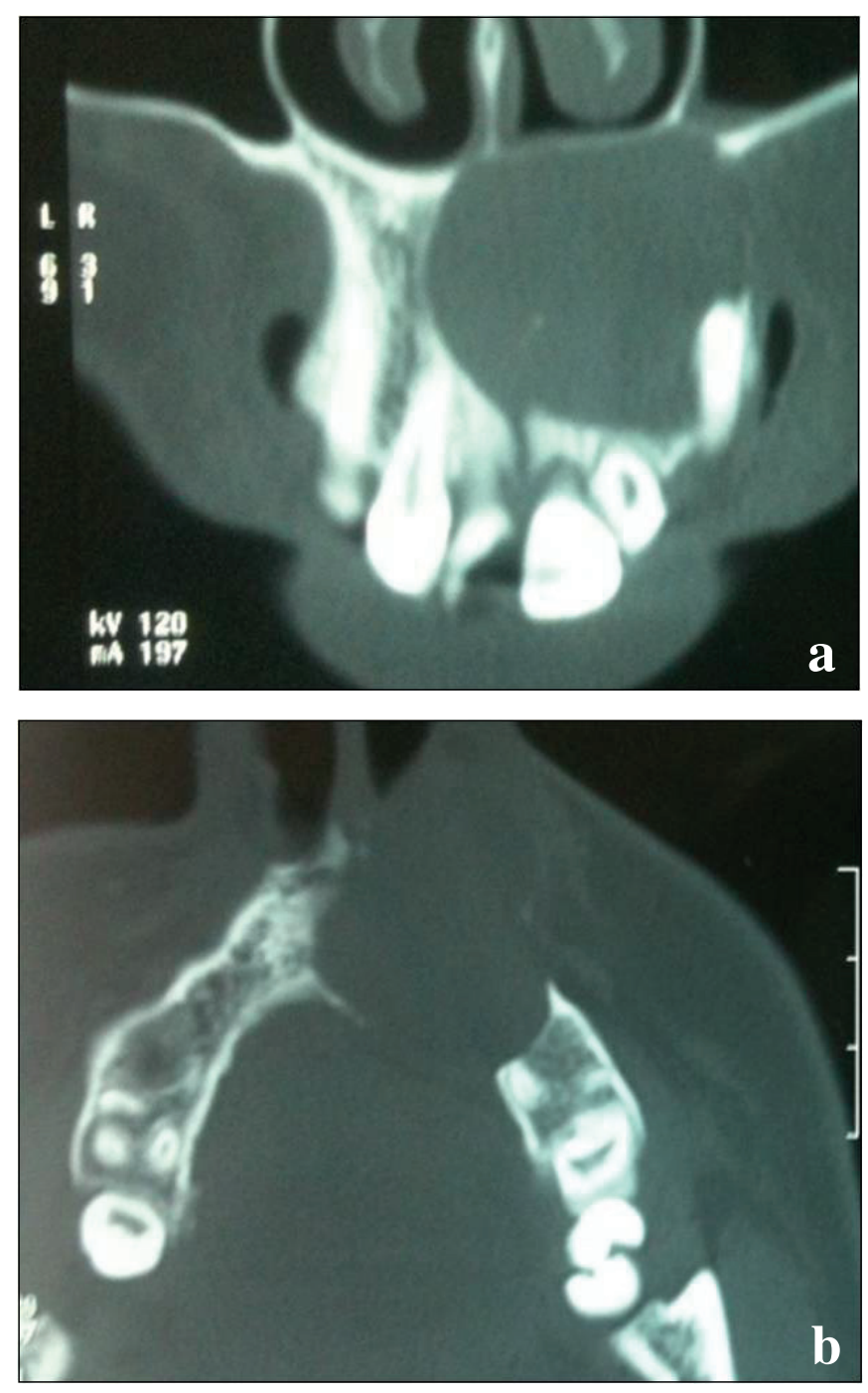

Fig. 4a et 4b. Dentascan (a. coupe axiale ; b. coupe frontale) : lésion hypodense bien limitée qui lyse les corticales interne et externe et qui présente des rapports étroits avec les fosses nasales et le sinus maxillaire gauche.

Fig. $4 a$ and $4 b$. Dentascan (a. axial slice; b. coronal slice): hypodense lesion well defined that breaks the internal and external corticals and who has intimate relations with nasal fossa and left maxillary sinus.

Le traitement a comporté une kystectomie avec extraction de 43, 44, 45 et 46. L'examen anatomopathologique a conclu qu'il s'agissait d'un kératokyste type orthokératosique (Fig. 13). $\mathrm{Au} 3^{\mathrm{e}}$ mois postopératoire, le contrôle clinique et radiologique a montré une bonne cicatrisation muqueuse et un début de cicatrisation osseuse (Fig. 14 et 15).

\section{Discussion}

Dans la classification de l'0MS de 1992, les kératokystes étaient classés parmi les kystes épithéliaux des maxillaires,

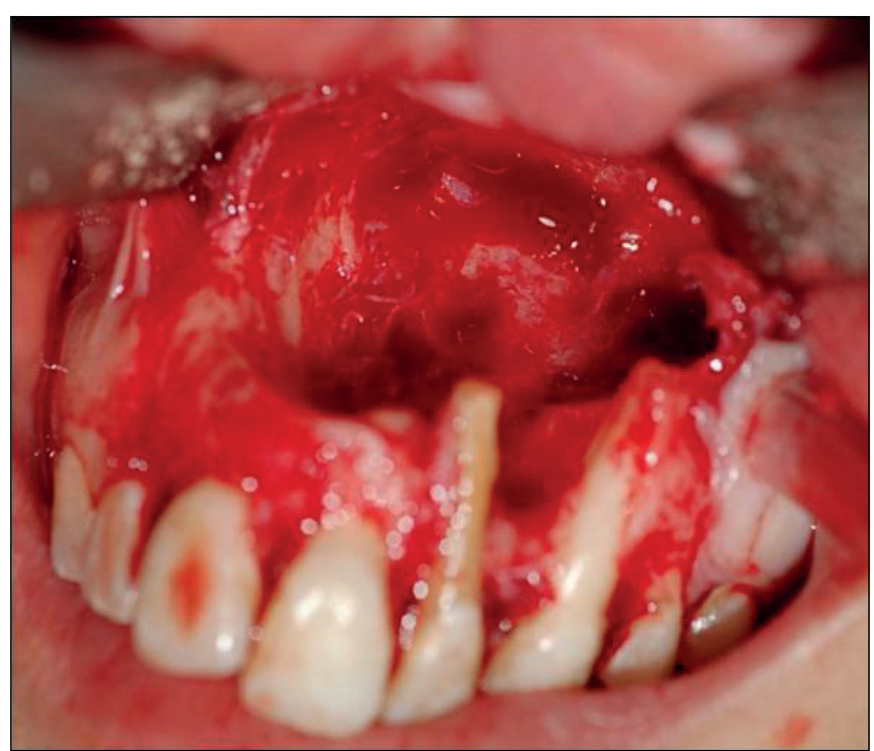

Fig. 5. Vue endobuccale : site opératoire après énucléation du kyste et résection apicale sur 11, 21 et 22 sur lesquelles on a effectué au préalable un traitement canalaire.

Fig. 5. Intraoral view: site operating after cystic enucleation and apical resection on 11, 21 and 22 having previously underwent endodontic treatment.

d'origine odontogène, dûs à une anomalie de développement [1] Dans la classification de 2005, sa variante parakératosique a été classée comme tumeur odontogène kératokystique (TOK). Elle est définie comme une tumeur bénigne uni ou multiloculaire, intra-osseuse, d'origine odontogène, dont la cavité est bordée par un épithélium pavimenteux stratifié parakératinisé ; cette tumeur a un potentiel agressif et infiltrant [2-5]. La TOK semble dériver de la lame dentaire, ou de ses vestiges, ou de l'assise basale de l'épithélium buccal. Dans 4 à $5 \%$ des cas, elle est associée à la naevomatose basocellulaire ou syndrome de Gorlin-Goltz. Une étude génétique systématique doit être réalisée pour tout kératokyste apparaissant avant l'âge de 20 ans $[6,7]$.

Cliniquement, le kyste peut se révéler par des douleurs, une tuméfaction intéressant un des maxillaires, parfois associée à un déplacement des dents et/ou à une perte de la sensibilité pulpaire (cas 1). Radiologiquement, la lésion se présente sous la forme d'une image radioclaire, unique ou multiloculaire, à contours nets, parfois associée à une dent incluse (cas 1);

Histologiquement, on retrouve un épithélium malpighien pluristratifié, d'épaisseur régulière, kératinisé avec une para ou orthokératose en surface [8].

Le diagnostic différentiel se fait avec toutes les lésions radioclaires uni ou multiloculaires des maxillaires [9] :

- le kyste inflammatoire : une dent mortifiée est à l'origine de ce kyste alors qu'avec le kératokyste, ce sont le déplacement important des dents et/ou la compression qui peuvent induire une perte de sensibilité de la pulpe (cas 1); 


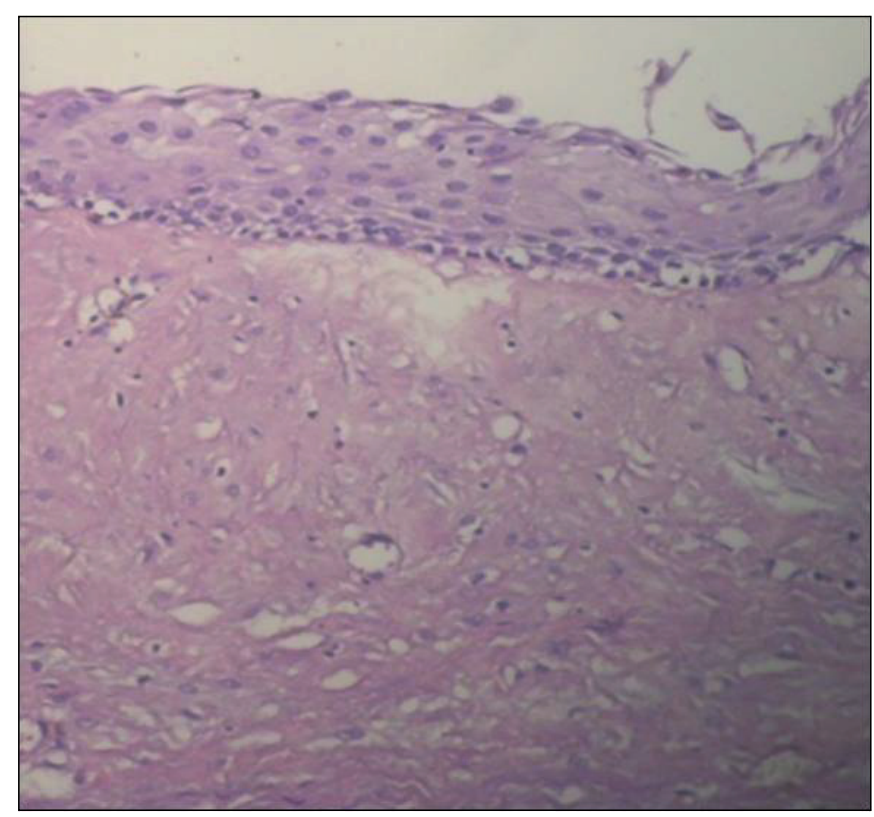

Fig. 6. Aspect microscopique de la paroi kystique : épithélium malpighien pluristratifié, d'épaisseur régulière, comportant 5 à 9 couches de cellules, avec une parakératose de surface.

Fig. 6. Microscopic aspect of the cystic wall: multilayered squamous epithelium, with 5 to 9 cell layers and a regular thickness with parakeratosis on the surface.

- le kyste dentigère : il se développe autour de la couronne d'une dent incluse, alors qu'avec le kératokyste, c'est le développement important du kyste qui va refouler une dent et empêcher son éruption (cas 2). Dans le cas où le kératokyste se développe lors de l'édification radiculaire, on observe une déformation de la racine (incurvation ou courbure) (cas $n^{\circ} 2$ ). Lorsqu'il se développe en juxtaposition à un élément dentaire en formation, il peut causer la dilacération des racines. Par contre, si le kyste se développe après l'éruption des dents, il peut entrainer des déplacements et des malpositions dentaires (cas 1 et 2 );

- l'améloblastome : une image polygéodique en bulles de savon oriente vers le diagnostic d'améloblastome ou de kératokyste. De plus, pour une lésion siégeant dans l'angle mandibulaire ou le ramus, ces deux entités sont systématiquement évoquées car c'est leur localisation préférentielle ;

- le fibrome : il correspond à une lésion radiotransparente sans calcifications, bien limitée, à prédominance mandibulaire postérieure, associée dans les $3 / 4$ des cas à une dent incluse.

Bien qu'ayant une structure d'aspect bénin, le kératokyste odontogène est considéré comme une tumeur agressive avec une tendance élevée à la récidive. Dans la littérature, on retrouve différentes propositions thérapeutiques allant des

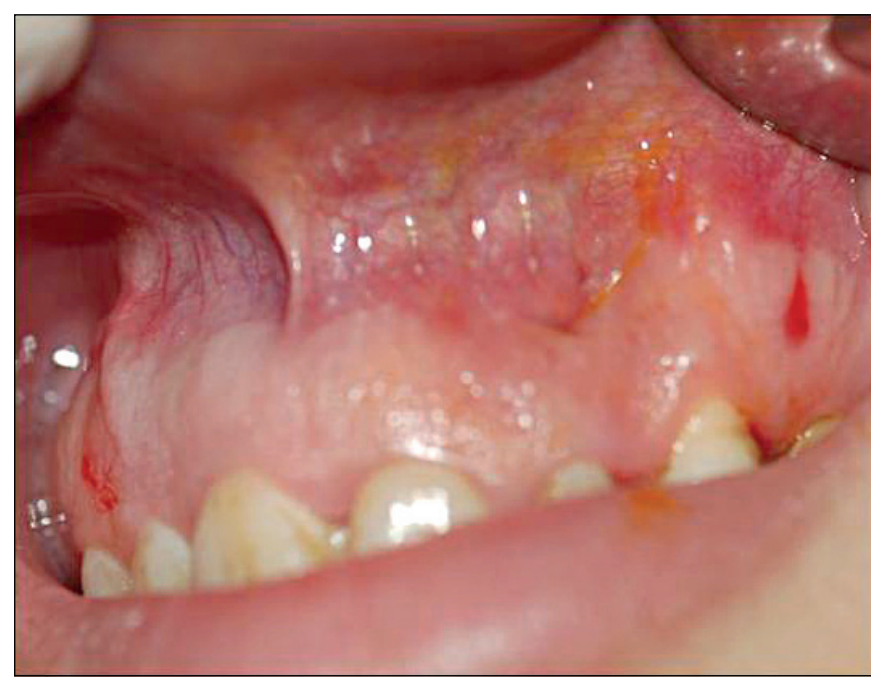

Fig. 7. Contrôle clinique $\left(3^{\mathrm{e}}\right.$ mois postopératoire $)$ : bonne cicatrisation muqueuse.

Fig. 7. Clinical control ( $3^{\text {rd }}$ postoperative month): good mucosal healing.

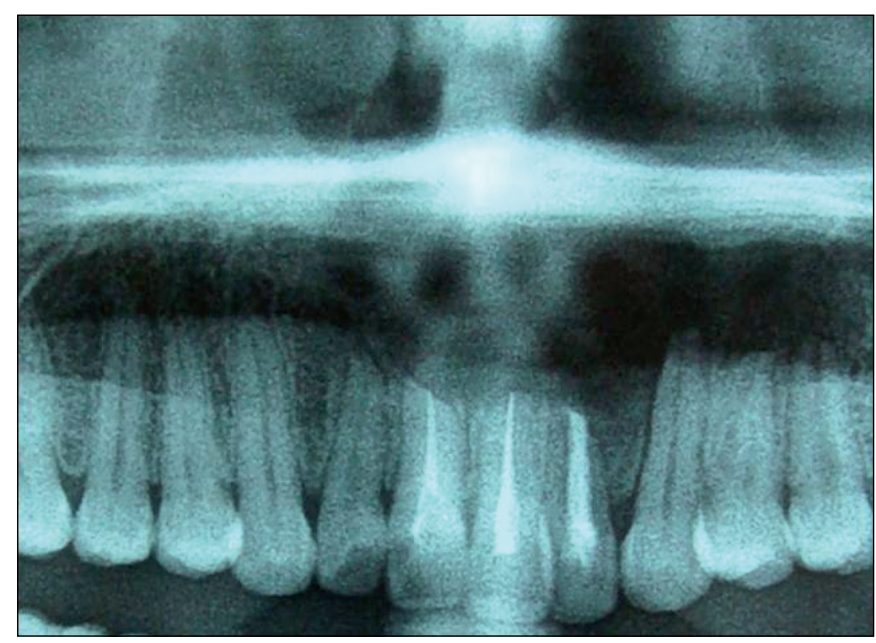

Fig. 8. Radiographie panoramique (1 an postopératoire). Fig. 8. Panoramic radiograph (1 postoperative year).

traitements conservateurs (énucléation avec ou sans curetage, décompression, marsupialisation) à des traitements plus ou moins agressifs (application de la solution de Carnoy, cryothérapie avec de l'azote liquide, ostéotomie périphérique avec un instrument rotatif, résection $[5,8,10]$. Tous ces traitements cherchent à diminuer les récidives et à éviter les séquelles fonctionnelles et esthétiques.

La radiothérapie a été utilisée ponctuellement pour le traitement des kératokystes récidivants, mais il y a un risque de dégénérescence maligne des lésions. La chimiothérapie n'est pas indiquée dans le traitement des kératokystes récidivants [10]. 


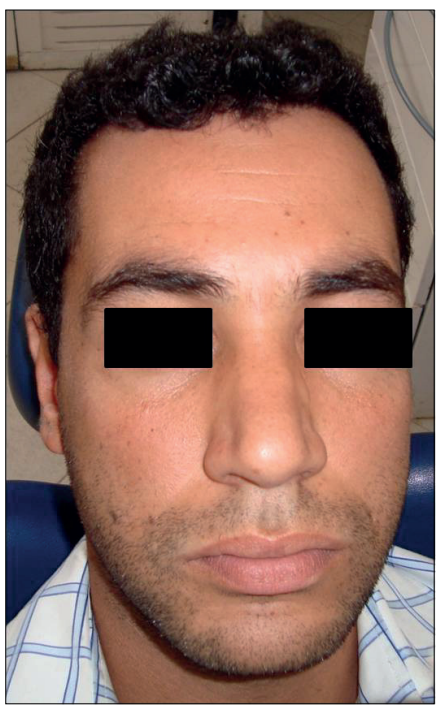

Fig. 9. Vue exobuccale : discrète tuméfaction génienne basse droite. Fig. 9. Exobuccal view: low left laterofacial swelling.

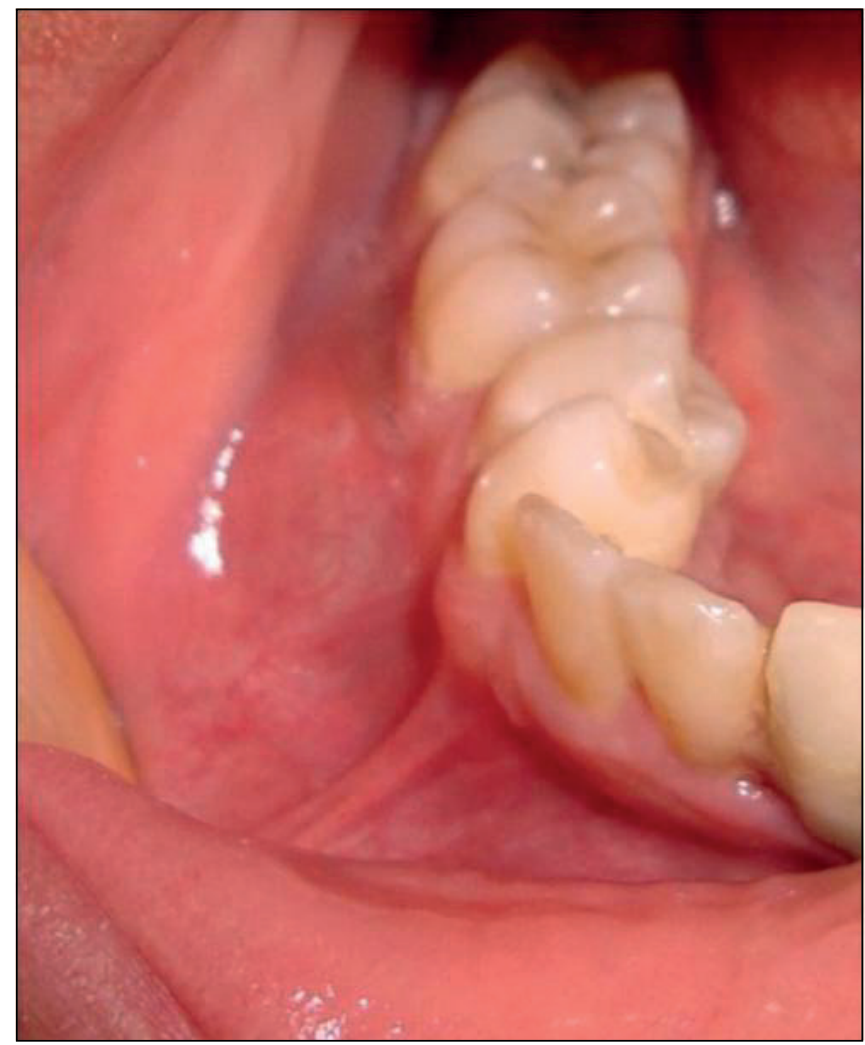

Fig. 10. Vue endobuccale : comblement vestibulaire et linguoposition de 44 et 45.

Fig. 10. Intraoral view: vestibular swelling and lingual displacement of 44 and 45.

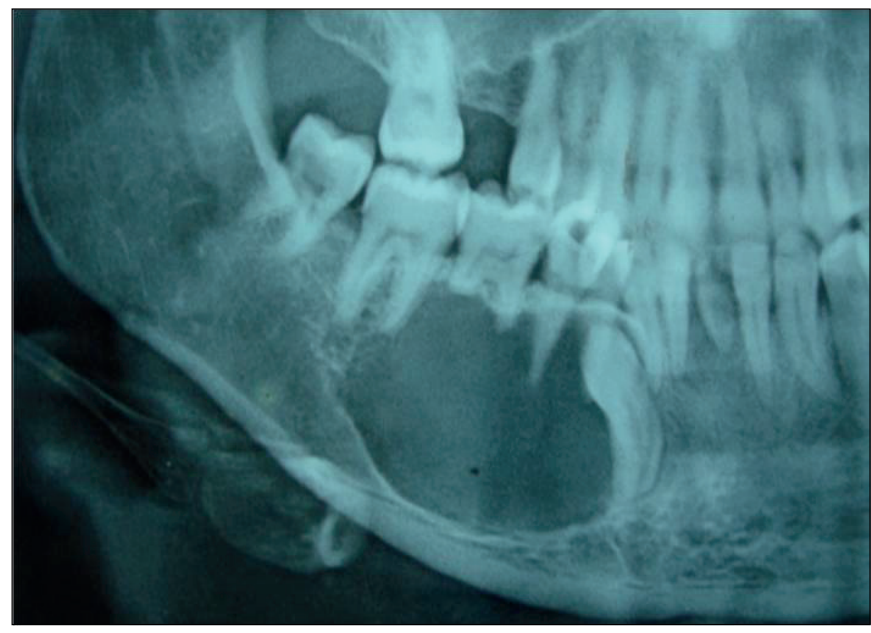

Fig. 11. Radiographie panoramique : image radioclaire, d'aspect kystique, bien limitée, avec 43 incluse et rhizalyse de 2 racines de 46 . Fig. 11. Panoramic radiograph: image-like radiolucent cystic, well limited, with 43 included and resorption of the 2 roots of 46 .
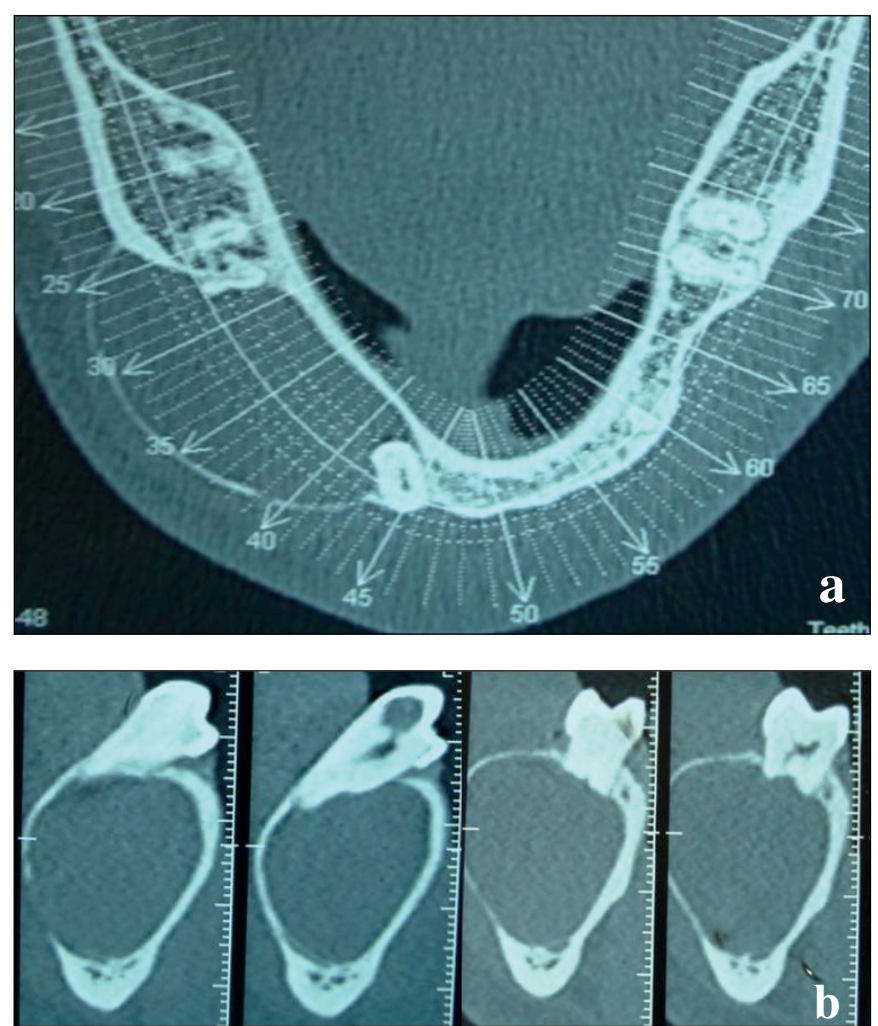

Fig. 12a et 12b. Dentascan. a (coupe axiale) : lésion hypodense, bien limitée soufflant la corticale externe $; b$ (coupe coronale oblique) : la lésion a refoulé le nerf alvéolaire inférieur en bas et a entrainé une rhizalyze plus ou moins marquée sur 44,45 et 46 .

Fig. $12 a$ and 12b. Dentascan. a (axial slice): hypodense and well defined lesion that blew the external cortical; $b$ (oblique coronal slice): the lesion has repressed the inferior alveolar nerve down and led to a reduction of 44,45 and 46. 


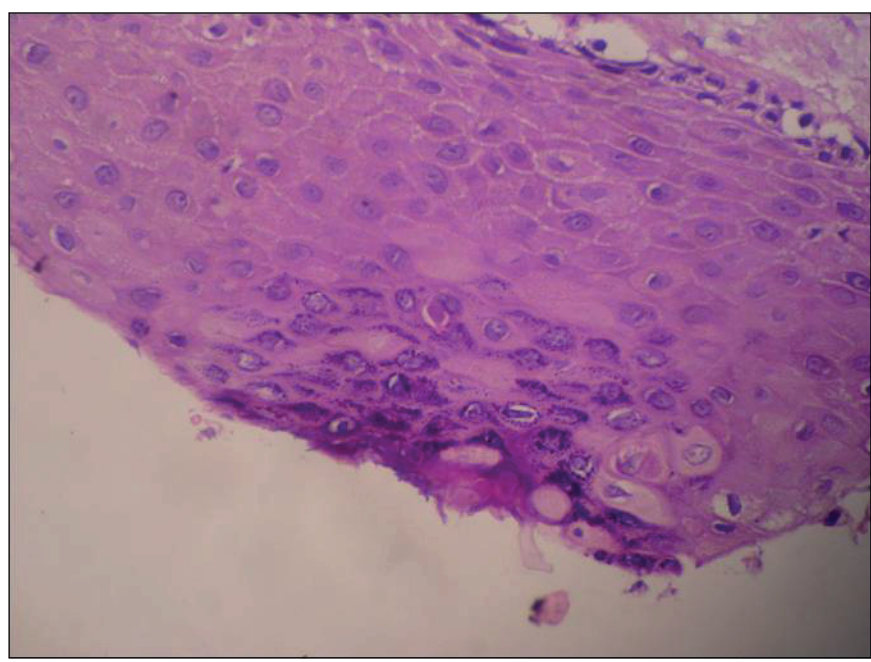

Fig. 13. Aspect microscopique : épithélium malpighien pluristratifié, d'épaisseur régulière, avec une orthokératose en surface.

Fig. 13. Microscopic aspect: multilayered squamous epithelium, with a regular thickness and orthokeratosis on the surface.

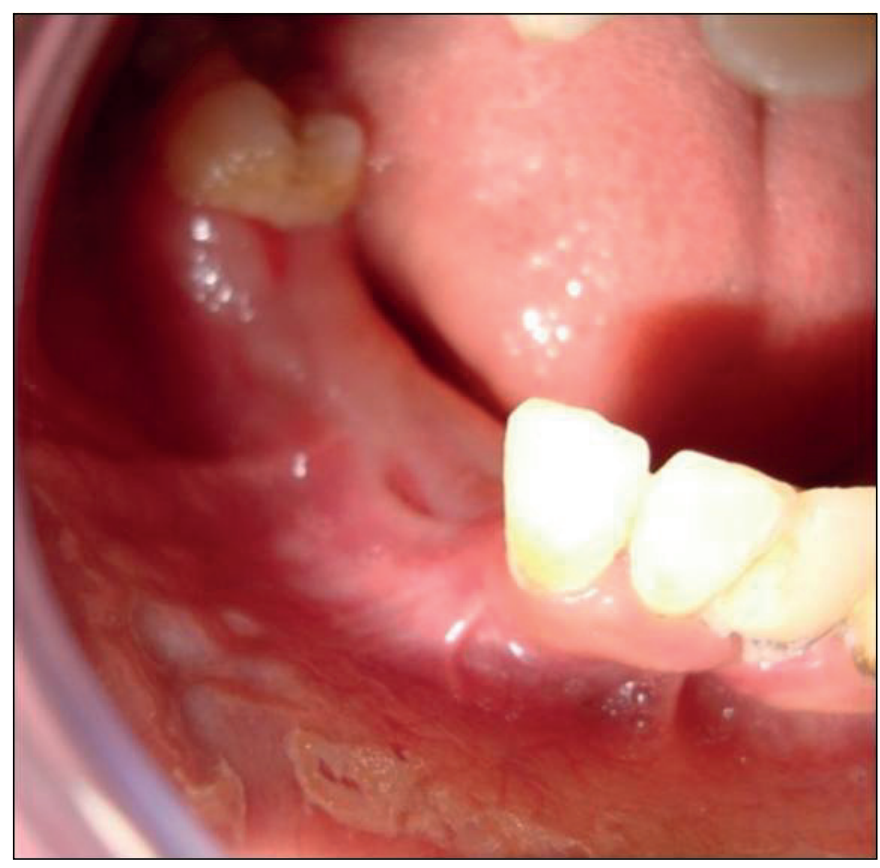

Fig. 14. Contrôle clinique $\left(3^{\mathrm{e}}\right.$ mois postopératoire) : bonne cicatrisation muqueuse.

Fig. 14. Clinical control ( $3^{\text {rd }}$ postoperative month): good mucosal healing.

Les kératokystes présentent un taux de récidives comparable à celui des améloblastomes. Selon certaines études, le taux de récidive peut atteindre $62 \%$ des cas [11]. La récidive des kératokystes serait plus élevée pour la localisation mandibulaire, lorsque le kératokyste est de type parakératosique, que

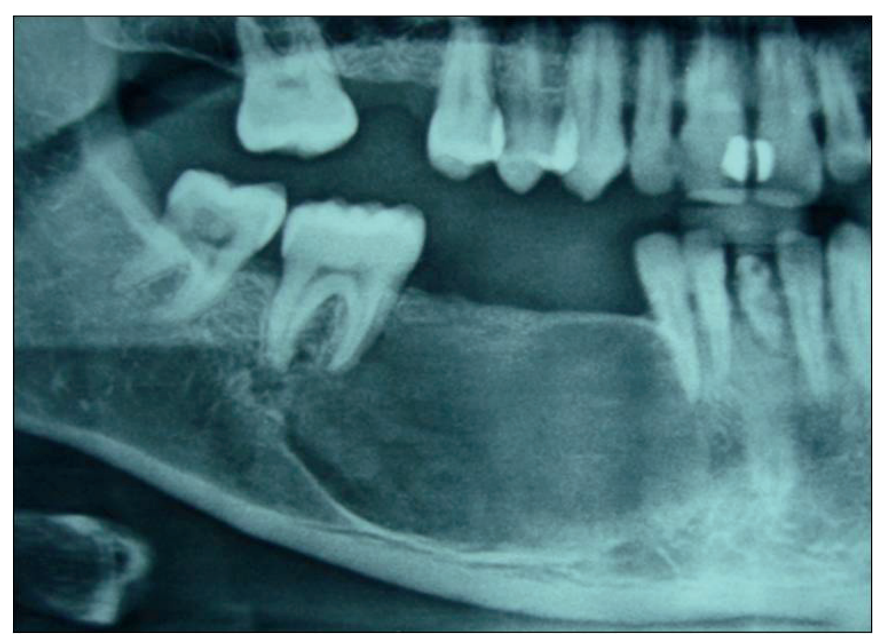

Fig. 15. Radiographie panoramique ( $3^{\mathrm{e}}$ mois postopératoire) : début de cicatrisation osseuse.

Fig. 15. Panoramic radiograph ( $3^{\text {rd }}$ postoperative month): early bone healing.

son aspect radiologique est multiloculaire et que l'exérèse a été réalisée en plusieurs fragments [12]. Enfin, des transformations améloblastiques (dans le contexte du syndrome de Gorlin-Goltz) et malignes (carcinomes épidermoïdes) ont été décrites [13], ce qui impose un suivi postopératoire à la fois clinique (tous les 6 mois) et radiologique (TDM après 18 mois). La durée du suivi fait l'objet de controverses. Crowley et al. ont montré que $25 \%$ des kératokystes avaient récidivé 9 ans après le traitement initial [14].

\section{Conclusion}

Le kératokyste odontogène présente une agressivité locorégionale et un risque de récidive plus important que les autres kystes des maxillaires. C'est pour ces raisons que le kératokyste est maintenant plutôt considéré comme une néoplasie kystique bénigne que comme un simple kyste odontogène.

Le diagnostic de certitude est basé sur la confrontation d'éléments cliniques, radiologiques et anatomopathologiques. Le traitement des kératokystes odontogènes reste essentiellement chirurgical mais les choix thérapeutiques sont multiples, variés et font l'objet de controverses.

\section{Conflits d'intérêt : aucun}

\section{Références}

1. Bornstein MM, Filippi A, Altermatt HJ, Lambrecht JT, Buser D. Kératokyste odontogène : kyste odontogène ou tumeur bénigne? Rev Mens Suisse Odontostomatol 2005;115:123-8. 
2. Hyun HK, Hong SD, Kim J. Recurrent keratocystic odontogenic tumor in the mandible: a case report and literature review. Oral Surg Oral Med Oral Pathol Oral Radiol Endod 2009;108: e7-10.

3. Shetty DC, Urs AB, Godhi S, Gupta S. Classifying odontogenic keratocysts as benign cystic neoplasms: a molecular insight into its aggressiveness. J Maxillofac Oral Surg 2010;9:30-4.

4. Bhagavandas Rai A, Charan Babu HS, Joshi M. A radiolucent lesion crossing the midline in maxilla: a rare presentation of odontogenic keratocyst in young patient. J Maxillofac Oral Surg 2010;9:102-4.

5. Le Toux G, Ales R-P, Mounier C. Approche chirurgicale des kératokystes odontogénes : à propos de deux cas cliniques. Med Buccale Chir Buccale 2001;7:33-41.

6. August M, Faquin WC, Troulis MJ, Kaban LB. Dedifferentiation of odontogenic keratocyst epithelium after cyst decompression. J Oral Maxillofac Surg 2003;61:678-83.

7. Anehosur V. Gorlin's syndrome. Report of a case and management of cystic lesions. J Oral Maxillofac Surg 2009;8:184-7.

8. Kissi L, Benyahya I, Rifki A. Le kératokyste isolé : à propos d'un cas. J Ordre Dent Quebec 2006;43:321-8.
9. Perrin JP, Mercier JM, SchmidtJ, Piot B. Très grands kératokystes mandibulaires : mise au point. Rev Stomatol Chir Maxillofac 2002;103:207-20.

10. Fotso J, Legrand W, Magremanne M. Recurrent maxillo-mandibular odontogenic keratocysts: prevention and treatment. J Rev Med Brux 2009;30:515-9.

11. González-Alva P, Tanaka A, Oku Y, Yoshizawa D, Itoh S, Sakashita H, Ide F, Tajima Y, Kusama K. Keratocystic odontogenic tumor: a retrospective study of 183 cases. J Oral Sci 2008;50:205-12.

12. Chemli H, Dhouib M, Karray F, Abdelmoula M. Les facteurs de risque de récidive des kératokystes odontogéniques des maxillaires. Rev Stomatol Chir Maxillofac 2010;111:189-92.

13. Zhang LL, Yang R, Zhang L, Li W, MacDonald-Jankowski D, Poh CF. Dentigerous cyst: a retrospective clinicopathological analysis of 2082 dentigerous cysts in British Columbia, Canada. Int J Oral Maxillofac Surg 2010;39:878-82.

14. Crowley TE, Kaugars GE, Gunsolley JC. Odontogenic keratocysts: a clinical and histologic comparison of parakeratin and orthokeratin variants. J Oral Maxillofac Surg 1992;50:22-6. 